\title{
Conference registration: how people react to a deadline
}

To the Editor - The 'Statphys' conferences on statistical physics take place every three years on a different continent. The number of participants can fluctuate greatly from conference to conference, and it is important that the organizers have an idea of this number as early as possible.

Statphys 23 took place in Genova, Italy, on 9-13 July 2007 (ref. 1). The registration website was activated around the end of January, with the deadline for registration and abstract submission on 31 March. The influx of registrations showed, from the outset, clear linear behaviour. The problem was, however, that the extrapolation of this linear behaviour to the deadline gave a very low number of expected participants. Clearly people tend to register late and one should expect a steepening of the distribution as the deadline approaches but by how much? Is it possible to predict accurately the final number of registrants?

In the case of another meeting - the International Conference on Electronic Properties of Two-dimensional Systems ${ }^{2}$ (EP2DS 17), organized by Vittorio Pellegrini and Alessandro Tredicucci (who kindly gave us their data) and also in Genova but with an earlier deadline - registrations also followed the initial linear behaviour, but with a different slope as the total number of participants was smaller. We rescaled the slope and used these data to produce an expectation curve for Statphys registrations, enabling us to predict that the final number of registrations for Statphys should exceed 1,000 - a value which is about three times larger than that expected from a linear extrapolation. We then tracked the actual registrations for Statphys (Fig. 1), and they did indeed reproduce the expected behaviour accurately (the small mismatches corresponding to weekends).

The similarity of the two curves suggests that there may be a universal behaviour for the dynamics of registration, and points to the possibility of defining a general model to describe how people respond to a deadline. The simplest assumption in this respect is that the 'pressure' to register increases as the inverse of the remaining time to the deadline. The probability $p(t)$ to register at time $t$ is then $p(t)=C /\left(t-T^{\star}\right)$, where $T^{\star}$ is the deadline and the constant $C$ will be fixed by the total number of participants $N_{\text {tot }}$. The number of registrations at time $t$ is given by

$N(t)=C \int p\left(t^{\prime}\right) \mathrm{d} t^{\prime}=C \ln \left(T^{*} /\left(T^{*}-t\right)\right)$. This leads to a logarithmic singularity that can be regularized using the discreteness of each registration day. As can be seen in

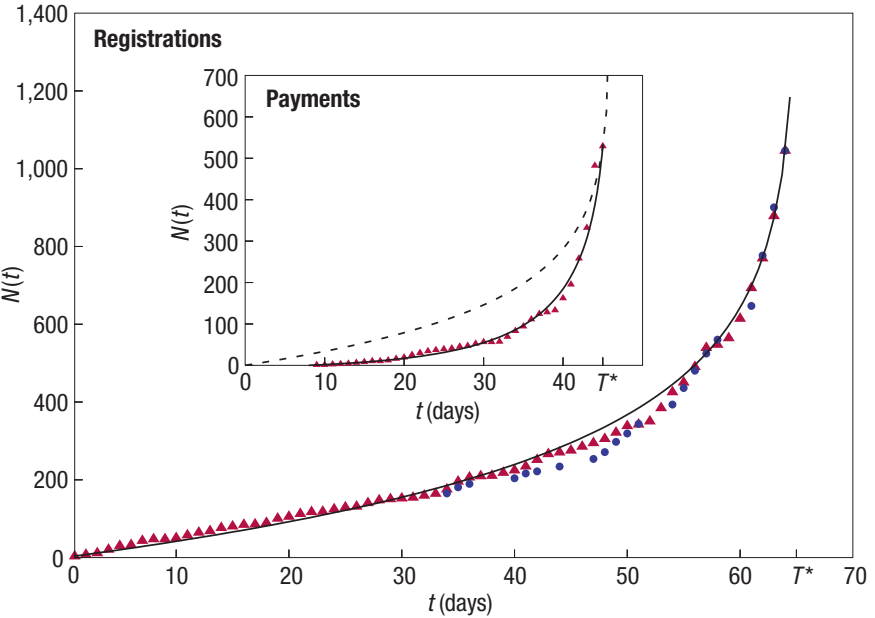

Figure 1 The distribution of registrations is shown for Statphys 23 (red triangles), up to the main deadline for abstract submission ( $T^{\star}$ ), and for the EP2DS 17 conference (blue circles), rescaled with respect to the total number of participants; the solid line corresponds to a simple model in which the pressure to register is inversely proportional to the time left before the deadline. The level of agreement between the data for the two conferences and the model suggests that there is a simple universal behaviour in response to a deadline. The inset shows the distribution in time of payment of the conference fee (credit-card payments only): the distribution is more peaked towards the deadline because, although registration is reversible, payment is irreversible. The simple model (dashed line) is not accurate in this case, and it is necessary to include an exponential utility function (solid line).

Fig. 1, this simple model fits the observed behaviour extremely well.

In principle, a term $\left(N^{\prime}-N(t)\right)$ should be included in the integral, where $N^{\prime}$ is the total number of people who have considered registering. The fact that the fit is so good without this term implies, in our opinion, that $N^{\prime}>N_{\text {tot }}$ : then $\left(N^{\prime}-N(t)\right)$ is essentially constant and can be absorbed in the constant $C$.

The model only assumes that the probability to register is uniform for the whole of the remaining time. In this respect, there is no real tendency to postpone the registration towards the deadline. This might seem curious, but note that the data refer only to the registration - payment could be made at a later time. The insert in Fig. 1 shows the distribution of payment times (only those made by credit card, for which the time can be precisely recorded; these are about half of the total registrations): the simple model does not describe these data. In fact, here it is natural to introduce a 'utility function' to describe the tendency to postpone the payment, if not the registration, until closer to the deadline. In analogy with the Boltzmann factor, this function can be modelled as $\exp \left(-\left(T^{*}-t\right) / \tau\right)$, where $\tau$ represents a characteristic time for the pressure to postpone. With this modification, the model fits the payment data rather well, as shown in the inset to Fig. 1, and $\tau$ is estimated to be 19.5 days.

People's behaviour around a deadline does indeed seem to be universal. If the action is reversible (as is simple registration), the pressure to do it is inversely proportional to the available time before the deadline. For an irreversible action (such as payment), there is a tendency to postpone it until even closer to the deadline, which can be described by a utility function. The rule of thumb to guess the final number of registrants is to consider the extrapolation of the initial linear behaviour and multiply it by three - a result that may be useful for organizers of future events. Finally, we note that one could consider the response to a deadline also from the point of view of microscopic models of human dynamics ${ }^{3}$.

\footnotetext{
Valentina Alfi, ${ }^{1,2}$, Giorgio Parisi ${ }^{1}$ and Luciano Pietronero ${ }^{1,3}$

${ }^{1}$ Dipartimento di Fisica, Università di Roma Sapienza, Roma, Italy

${ }^{2}$ Centro Studi e Ricerche E. Fermi, Roma, Italy ${ }^{3}$ Istituto dei Sistemi Complessi, CNR, Roma, Italy e-mail: luciano.pietronero@roma1.infn.it
}

References

1. www.statphys23.org

2. www.ep2ds-mss.infm.it

3. Barabási, A.-L. Nature 435, 207-211 (2005). 